BULLETIN OF THE

AMERICAN MATHEMATICAL SOCIETY

Volume 79, Number 4, July 1973

\title{
A RESTRICTION ON THE PARAMETERS OF A SUBQUADRANGLE
}

\author{
BY STANLEY E. PAYNE \\ Communicated by Gian-Carlo Rota, January 8, 1973
}

1. Introduction. A generalized quadrangle of order $(s, t)$ is a finite incidence plane $P$ with $v_{1}=(1+t)(1+s t)$ lines, $v_{2}=(1+s)(1+s t)$ points, and a symmetric incidence relation I satisfying the following axioms (cf. [1]):

I-1. No two lines of $P$ are incident with two points in common.

I-2. If $x$ is a point of $P$ and $L$ is a line of $P$ such that $x \backslash L$ (i.e., $x$ is not incident with $L)$, then there is a unique pair $\left(x^{\prime}, L^{\prime}\right)$ consisting of a point and line, respectively, such that $x \mathrm{I} L^{\prime}, x^{\prime} \mathrm{I} L^{\prime}$, and $x^{\prime} \mathrm{I} L$.

I-3. Each line (point) is incident with $1+s$ points $(1+t$ lines).

Throughout this note, $P$ will denote a generalized quadrangle of order $(s, t)$ and $Q$ a subquadrangle of $P$ of order $\left(s^{\prime}, t^{\prime}\right)$ with $1 \leqq s^{\prime} \leqq s, 1 \leqq t^{\prime} \leqq t$. In [4], Thas gives a number of restrictions on $s^{\prime}$ and $t^{\prime}$ in terms of $s$ and $t$. Two of them are as follows in case $s^{\prime}<s$ and $t^{\prime}<t$ :

(1) $s^{\prime}\left(t^{\prime}\right)^{2}<s t$ and $t^{\prime}\left(s^{\prime}\right)^{2}<s t$.

(2) If $t=s$ and $t^{\prime}=s^{\prime} \geqq 13$, then $s^{2}>3\left(s^{\prime}\right)^{3}$.

It is the purpose of this note to give the following improvement of (1) and (2), which is a "best possible" result in the sense that, for each prime power $s^{\prime}$, the case $s=\left(s^{\prime}\right)^{2}\left(s=t, s^{\prime}=t^{\prime}\right)$ does arise.

THEOREM. With $s, s^{\prime}, t, t^{\prime}$ as above, it must be that

$$
\text { (a) } s \geqq s^{\prime} t^{\prime} \text { or } s=s^{\prime} \quad \text { and dually } \quad \text { (b) } t \geqq s^{\prime} t^{\prime} \text { or } t=t^{\prime} \text {. }
$$

Thas examines rather thoroughly the case $s=s^{\prime}, t>t^{\prime}$, and we refer the reader to [4] for several results in this case.

2. Proof of the Theorem. Our proof of the Theorem is based on ideas of D. G. Higman and C. Sims, particularly as developed in [2] and [3].

Let $G$ be the graph whose vertices are the points of $P$ and whose edges are the pairs of noncollinear points of $P$. Let $A$ be the $(0,1)$ adjacency matrix of $G$ defined in terms of some fixed ordering of the vertices of $G$. Then $A$ is symmetric with characteristic roots $-s, t$, and $s^{2} t$. Partition the vertices of $G$ into two sets $\Delta_{1}$ and $\Delta_{2}$ as follows: $\Delta_{1}$ is the set of points of $Q ; \Delta_{2}$ is the set of points of $P$ not in $Q$. For convenience put

$$
n_{1}=\left|\Delta_{1}\right|=\left(1+s^{\prime}\right)\left(1+s^{\prime} t^{\prime}\right), \quad n_{2}=\left|\Delta_{2}\right|=(1+s)(1+s t)-n_{1} .
$$

AMS (MOS) subject classifications (1970). Primary 05B25; Secondary 15A42.

Key words and phrases. Finite generalized quadrangle, subquadrangle. 
For $1 \leqq i, j \leqq 2$, let $e_{i j}$ be the number of ordered pairs $(x, y)$ for which $x \in \Delta_{i}, y \in \Delta_{j}$, and $(x, y)$ is an edge of $G$. Let $\hat{A}$ be the $2 \times 2$ matrix whose $(i, j)$ entry is $e_{i j} / n_{i}$. According to the theorem of Sims quoted on p. 144 of [2], the characteristic roots of $\hat{A}$ must lie between $-s$ and $s^{2} t$.

A straightforward computation shows that

$$
\hat{A}=\left(\begin{array}{cc}
\left(s^{\prime}\right)^{2} t^{\prime} & s^{2} t-\left(s^{\prime}\right)^{2} t^{\prime} \\
\frac{n_{1}\left(s^{2} t-\left(s^{\prime}\right)^{2} t^{\prime}\right)}{n_{2}} & s^{2} t-\frac{n_{1}\left(s^{2} t-\left(s^{\prime}\right)^{2} t^{\prime}\right)}{n_{2}}
\end{array}\right) .
$$

It is easy to see that $s^{2} t$ is a root of $\hat{A}$, so that $\operatorname{tr}(\hat{A})-s^{2} t=\left(s^{\prime}\right)^{2} t^{\prime}-$ $\left(n_{1}\left(s^{2} t-\left(s^{\prime}\right)^{2} t^{\prime}\right)\right) / n_{2}=r$ is the other root. The condition that $-s \leqq r$ is then easily shown to be equivalent to

$$
0 \leqq\left(s-s^{\prime} t^{\prime}\right)\left(s-s^{\prime}\right) .
$$

COROLlARY. If a generalized quadrangle $P$ of order $s$ (i.e., $s=t$ ) has a subquadrangle $Q$ of order $s^{\prime}$, then $s \geqq\left(s^{\prime}\right)^{2}$.

\section{BIBLIOGRAPHY}

1. W. Feit and G. Higman, The nonexistence of certain generalized polygons, J. Algebra 1 (1964), 114-131. MR 30 \# 1189.

2. M. D. Hestenes and D. G. Higman, Rank 3 groups and strongly regular graphs, SIAM-AMS Proc., vol. 4, Amer. Math. Soc., Providence, R.I., 1971, pp. 141-159.

3. D. G. Higman, Partial geometries, generalized quadrangles and strongly regular graphs, Atti del convegno di geometria combinatoria e sua applicazioni, Perugia, 1971.

4. J. A. Thas, 4-gonal subconfigurations of a given 4-gonal configuration (unpublished manuscript).

Department of Mathematics, Miami University, Oxford, Ohio 45056 